\title{
Criminologie
}

\section{Prévention des gangs de rue à Montréal : autour d'un projet de médiation urbaine}

\section{Gilles Sénécal, Léa Méthé Myrand et Amélie Dubé}

Volume 43, numéro 1, printemps-été 2010

Les jeunes et la rue

URI : https://id.erudit.org/iderudit/044052ar

DOI : https://doi.org/10.7202/044052ar

Aller au sommaire du numéro

\section{Éditeur(s)}

Les Presses de l'Université de Montréal

ISSN

0316-0041 (imprimé)

1492-1367 (numérique)

Découvrir la revue

Citer cet article

Sénécal, G., Méthé Myrand, L. \& Dubé, A. (2010). Prévention des gangs de rue à Montréal : autour d'un projet de médiation urbaine. Criminologie, 43(1),

91-114. https://doi.org/10.7202/044052ar
Résumé de l'article

Le présent article analyse le processus de relance d'un projet de médiation urbaine destiné spécifiquement aux jeunes Noirs du quartier de la Petite-Bourgogne à Montréal. Ce projet nommé BUMP (Burgundy Urban Mediation Project) s'inscrit dans les orientations définies au Québec en matière de prévention des gangs de rue et qui font appel à la concertation des partenaires sociaux à l'intérieur du quartier. L'analyse du processus a mis en lumière les conditions particulières du quartier et des modèles organisationnels des réseaux sociaux. Notre étude dégage les termes de la transaction sociale survenue entre les partenaires sociaux et les représentants des organismes de la communauté noire pour qu'un tel projet de médiation urbaine puisse être relancé. Elle s'intéresse aux obstacles et aux facteurs favorables à une telle démarche participative et partenariale. Elle conclut sur le rôle des représentants des communautés marginalisées dans les instances de concertation de quartier.
Ce document est protégé par la loi sur le droit d'auteur. L'utilisation des services d’Érudit (y compris la reproduction) est assujettie à sa politique d'utilisation que vous pouvez consulter en ligne.

https://apropos.erudit.org/fr/usagers/politique-dutilisation/ 


\title{
Prévention des gangs de rue à Montréal : autour d'un projet de médiation urbaine
}

\author{
Gilles Sénécal \\ Professeur \\ Institut national de la recherche scientifique \\ INRS Centre Urbanisation Culture Société \\ gilles.senecal@ucs.inrs.ca \\ Léa Méthé Myrand \\ Diplômée à la maîtrise \\ Programme en études urbaines \\ Institut national de la recherche scientifique \\ INRS Centre Urbanisation Culture Société \\ Léa.méthemyrand@ucs.inrs.ca \\ Amélie Dubé \\ Assistante de recherche \\ amedube@yahoo.ca
}

RÉSUMÉ - Le présent article analyse le processus de relance d'un projet de médiation urbaine destiné spécifiquement aux jeunes Noirs du quartier de la Petite-Bourgogne à Montréal. Ce projet nommé BUMP (Burgundy Urban Mediation Project) s'inscrit dans les orientations définies au Québec en matière de prévention des gangs de rue et qui font appel à la concertation des partenaires sociaux à l'intérieur du quartier. L'analyse du processus a mis en lumière les conditions particulières du quartier et des modèles organisationnels des réseaux sociaux. Notre étude dégage les termes de la transaction sociale survenue entre les partenaires sociaux et les représentants des organismes de la communauté noire pour qu'un tel projet de médiation urbaine puisse être relancé. Elle s'intéresse aux obstacles et aux facteurs favorables à une telle démarche participative et partenariale. Elle conclut sur le rôle des représentants des communautés marginalisées dans les instances de concertation de quartier.

MOTS-CLÉS - Gangs de rue, médiation urbaine, quartier, transaction sociale, PetiteBourgogne, Montréal. 


\section{Introduction ${ }^{1}$}

Plusieurs projets de médiation urbaine auprès des jeunes ont été lancés ces dernières années dans une optique de prévention de la criminalité juvénile et de lutte aux gangs de rue. Un de ces projets visait plus particulièrement les jeunes de la communauté noire du quartier de la Petite-Bourgogne de Montréal. Démarrées en 2004, ses activités ont été interrompues en 2006. Les efforts pour sa relance ont mobilisé l'ensemble des acteurs sociaux du quartier, en particulier ceux qui participent au processus de concertation reconnu par le programme de l'Initiative montréalaise de soutien au développement social local, c'està-dire celui qu'anime la Table de concertation intersectorielle de quartier (ci-après nommée la Table), dans ce cas-ci la Coalition de la PetiteBourgogne.

Le problème observé en 2007 était justement l'arrimage difficile entre les institutions publiques et les acteurs communautaires confondus, reconnus comme les partenaires des processus de concertation, et les organisations de la communauté noire. Les discussions sur la relance du projet nommé Burgundy Urban Mediation Project (BUMP) ont révélé des divergences profondes quant au modèle organisationnel et au mode de fonctionnement de l'organisme chargé d'administrer le programme financé par la Ville de Montréal (Ville de Montréal, 2006). Elles ont fourni l'occasion d'observer l'élaboration d'une approche sociale et communautaire de prévention du phénomène des gangs de rue menée par les acteurs sociaux qui travaillent à l'intérieur du processus de concertation de quartier.

Le processus de relance mettait en scène une Table de quartier et des représentants de la communauté noire. La Table avait pour mandat de développer la prise en charge des problèmes par les communautés qui les vivaient; ce que revendiquaient précisément les représentants de la communauté noire. En ce sens, l'observation du processus a permis de comprendre les difficultés de déléguer aux acteurs communautaires un problème aussi sensible et complexe que celui des gangs de rue. À ce propos, il n'est pas inutile de rappeler que le Plan d'intervention québécois sur les gangs de rue privilégie l'approche territorialisée et

1. Gilles Sénécal remercie le Conseil de recherches en sciences humaines du Canada pour sa contribution financière. Les auteurs souhaitent remercier également les évaluateurs anonymes pour leurs commentaires. Ils expriment enfin toute leur gratitude aux personnes qui ont accepté de leur accorder une entrevue dans le cadre de cette étude. 
communautaire, d'autant plus qu'il y est reconnu que «certaines communautés culturelles vivent de façon toute particulière la problématique» (Gouvernement du Québec, 2007: 9). Cette délégation aux acteurs locaux de la fonction de concertation pose le défi, comme c'était le cas dans la Petite-Bourgogne, non seulement de rejoindre les organismes ou les représentants des communautés concernées, mais d'établir un programme d'intervention efficace et qui fonctionne selon les règles des programmes normés.

Notre étude porte précisément sur le processus de mobilisation et la mise en place d'une d'intervention dans un milieu précis. Notre objet n'est pas d'évaluer l'efficacité des interventions conduites par les médiateurs agissant auprès des jeunes. Notons que les activités du BUMP ont déjà fait l'objet d'une évaluation qui soulignait le caractère global et structurel des interventions des médiateurs, conduites en rupture avec les interventions conventionnelles et cloisonnées observées dans le passé, pour conclure que : «BUMP constitue en quelque sorte un service de médiation entre les individus et les institutions» (Tremblay et al., 2006: 40). L'institution dont il est question est celle du quartier, qui apparaît comme un espace public sur lequel les acteurs peuvent débattre et planifier des interventions portant sur une situation subie, celle du risque de progression des gangs de rue. Les exercices de concertation visent à produire de nouvelles situations à l'échelle du quartier, comme l'implantation du BUMP ou sa relance, par lesquelles les conflits sont traités et qu'une vision commune peut émerger. Notre étude propose de dégager les termes de la transaction sociale qui a permis de produire les conditions nécessaires à la relance du projet BUMP.

\section{De la régulation sociale à la transaction sociale}

La figure du quartier comme espace de régulation sociale procède d'une longue tradition en sociologie urbaine de même qu'en criminologie. D'une certaine façon, les explications qui privilégient les effets de quartier évoquent le rôle du milieu, prenant leur inspiration du côté de la sociologie durkheimienne (Milburn, 1999). L'école de Chicago en sociologie urbaine, avec des auteurs comme Burgess (1925), Wirth (1925 [1980]), Trasher (1927) et plus tard Whyte (1943), mettait de l'avant le terme d'aire sociale pour désigner l'espace occupé par les institutions de la communauté. On y comprenait la désorganisation sociale, notamment le phénomène des gangs de rue, comme le produit de culture ethnique 
(Tichit, 2003), d'une part, et comme l'effet de l'incapacité des institutions communautaires ethniques à réguler les faits sociaux à l'intérieur d'un territoire bien défini, d'autre part (Burgess, 1925; Block et al., 2007). Ainsi, sous les termes de milieu, d'aire sociale, de communauté ou de quartier, on désignait une forme territorialisée d'organisation sociale, qui était justifiée par une identité communautaire forte, mais qui en s'érodant faisait place à la désorganisation sociale. Ce rôle de régulation sociale exercé par les institutions communautaires était confronté à la mobilité personnelle des individus, qu'elle soit quotidienne (les navettages) ou résidentielle (le parcours de vie). Cela se traduisait par cette relation que l'on tentait d'établir entre les lieux de résidence, les sites des institutions communautaires et la localisation des crimes. Les fameux triangles de Burgess (de la communauté, de la mobilité et de la promiscuité) devaient démontrer que la désorganisation survient avec la fragmentation de l'espace urbain: les individus échappent, en se déplaçant à l'extérieur de l'aire sociale de la communauté, aux codes comportementaux de cette même communauté (Burgess, 1967: 152-153). La question de la distance entre le domicile et le lieu du crime, encore reprise aujourd'hui (Block et al., 2007), suscite la question de la capacité des organisations du quartier à prévenir les crimes pouvant être commis en son sein. Ce détour par l'école de Chicago n'est pas fortuit, car le phénomène de gangs de rue à Montréal est largement associé à des quartiers particuliers aux caractéristiques écologiques marquées: SaintMichel, Montréal-Nord et Petite-Bourgogne. Les solutions annoncées comportent souvent le recours à la mobilisation des acteurs communautaires. Ainsi, le Plan québécois de lutte aux gangs de rue reprend presque mot à mot le postulat de l'école de Chicago lorsqu'on y affirme que les facteurs d'adhésion à un gang tiennent «d'un environnement marqué par la désorganisation sociale» (Gouvernement du Québec, 2007: 8) et que la solution passe par l'intervention communautaire.

La prophétie de l'érosion des fonctions régulatrices et identitaires du quartier contenue dans les études de l'école de Chicago a été reprise, ces dernières années, par les tenants de la thèse de la fin des quartiers (Ascher, 1998; Chalas, 1997). La modernité avancée se caractériserait par la disparition des liens identitaires et des relations de proximité sous l'effet conjugué de l'hypermobilité des personnes et de la fragmentation de l'espace. Ce phénomène est aussi décrit par Giddens et Habermas sous les termes de la dé-localisation ou de déracinement des appartenances (Habermas, 1992: xxxiv; Giddens, 1994 : 29). Ceux-ci s'enten- 
dent toutefois pour reconnaître que des dispositifs de démocratie locale ou de délibération publique rendent possible la re-territorialisation: des liens de proximité s'établiraient avec «la construction d'appartenance et de rattachements communautaires propres»(Habermas, 1992: xxxiv), ce que Giddens qualifie d'engagement sur le plan local (Giddens, $1994: 154-155)$. La définition du quartier comme portion différenciée du territoire, chère aux géographes, fait place à celle du dispositif ou, autrement dit, au cadre d'action (Grefmeyer, 2007). Ce dispositif correspond à un autre type d'attachement, non plus basé sur la proximité spatiale du voisinage, mais plutôt sur les liens étroits que développent des représentants d'organismes fonctionnant en réseau et que certains auteurs nomment la proximité organisationnelle (Gilly et Lung, 2005). Un tel réseau est constitué d'acteurs sociaux qui sont compétents à délibérer des enjeux sociaux et éthiques, dont celui du vivre-ensemble (Stoessel-Ritz, 2005). Il ressort de cela que le quartier est reconnu comme un espace public à travers des réseaux sociaux institutionnalisés qui développent entre eux des relations de proximité. On peut, dès lors, prétendre que de tels réseaux de proximité, parfois même virtuels, parviennent à maintenir l'esprit de la communauté (Wellman et Leighton, 1979; Charbonneau, 2001; Wellman, 2001). En somme, le quartier devrait son existence, et sa reconnaissance, à un ensemble de réseaux sociaux animés par des personnes et des groupes qui font appel aux notions de solidarité et d'appartenance, afin de tenter de résoudre des problèmes et de reconstruire l'image du quartier. Par contre, cet espace réseau ne devrait sa capacité d'intervention qu'aux processus de démocratie citoyenne qu'il parvient à mettre en place. Il soumet les problèmes et les enjeux à des processus de délibération par lesquels des acteurs et des intervenants confrontent des intérêts souvent opposés et des valeurs différentes dans une recherche permanente de compromis plus ou moins équitables. Ces négociations autour du vivre-ensemble, incluant la détermination des règles de la vie sociale, concourent à une remise en question du paradigme de la désorganisation sociale et de la régulation. Les mécanismes de concertation, constitués à l'échelle du quartier, cherchent davantage à établir des convergences et des compromis, ce que nous définissons comme des transactions sociales. 


\section{La transaction sociale: une approche méthodologique compréhensive}

Notre approche méthodologique s'inscrit dans le courant général de l'interactionnisme en sociologie qui aborde les rapports établis entre des acteurs dans des situations données (Blumer, 1969; Goffman, 1974). Les situations qui nous intéressent sont construites par des acteurs qui agissent selon des intérêts, des enjeux, des valeurs ou des intentions qui leur sont propres (Mucchielli, 2004: 256). Lanalyse de la situation porte sur les interactions entre des acteurs sociaux et, dans ce cas-ci, ceux regroupés dans une Table de quartier et qui participent aux processus mis en place par cette même Table. La situation est observée à l'aide du récit qu'énoncent les acteurs sociaux lorsqu'ils sont invités à réfléchir sur une situation donnée. Elle est donc comprise d'abord et avant tout comme un cadre de l'action, dans lequel les interactions sociales se déroulent et par lequel une structure d'échange se manifeste. Les acteurs parviennent ainsi à identifier des problèmes et des enjeux, tout en défendant des valeurs et des principes qu'ils soumettent au jeu de l'échange, dans le but de négocier, de s'ajuster au contexte et d'établir ce que l'on nomme des transactions sociales. Le paradigme de la transaction sociale, qu'ont proposé Jean Rémy (1996) et Maurice Blanc (1992), entend éclairer les échanges se déroulant à l'intérieur de la société civile, à l'échelle de la vie quotidienne en ayant pour objet des problèmes marqués du sceau du changement et de l'innovation. L'analyse qu'il sous-tend voit l'articulation de ces échanges, qui impliquent une certaine réciprocité et une part de risque, comme le moteur des changements dans les dynamiques interpersonnelles et identitaires (Rémy, 1996). Les acteurs de la transaction sociale tentent de résoudre des conflits; ils acceptent tacitement de modifier leur jeu ou leur parcours afin de rendre possible la création d'un pont entre eux, sans toutefois prétendre satisfaire l'ensemble des parties (Blanc et al., 1998). En outre, de telles transactions se réalisent au contact des structures organisationnelles, celles de la société civile comme celles des institutions et des pouvoirs politiques; elles touchent, de multiples façons, la vie quotidienne des personnes concernées par de telles expériences relationnelles (Joseph et Quéré, 2003).

Le processus transactionnel amène les acteurs à s'adapter à la situation et au contexte, à développer de nouveaux réflexes identitaires, de nouvelles aptitudes par une séquence d'ajustements successifs. Dans un 
contexte d'interaction relativement encadrée et obligatoire comme celui d'une Table, la transaction sociale correspond à un "processus de socialisation ou d'intégration dans la société et d'apprentissage des compétences démocratiques» (Blanc et al., 1998: 219). Cet apprentissage conduit à la formation du quartier comme espace transactionnel, délimité spatialement, animé dans des délibérations successives sur les enjeux globaux et reconnu comme possédant une image, voire une identité particulière (Sénécal et al., 2008).

\section{Contexte et techniques d'enquête}

Le contexte de l'étude est d'un partenariat établi en 2003 entre les trois bailleurs du programme de l'Initiative montréalaise de soutien au développement social local (Ville de Montréal, Direction de la santé publique de Montréal et Centraide du Grand Montréal), la Coalition montréalaise des tables de quartiers (CMTQ) et une équipe de chercheurs afin d'établir les termes d'une étude portant sur les effets de concertation de quartiers et dont les orientations, notamment le cadre conceptuel et la méthodologie, ainsi que les questions et les hypothèses auxquelles il s'agissait de répondre, ont fait l'objet d'une démarche réflexive commune. La Coalition de la Petite-Bourgogne a accepté de participer à l'étude en proposant d'observer la situation de la relance du projet BUMP. Des observations et des entrevues sur ce cas ont commencé à l'été 2007 et des résultats préliminaires ont été discutés avec les représentants de la Table à l'automne suivant. Toutes les personnes ayant participé aux discussions entourant la relance du BUMP ont été rencontrées. Le contenu des entretiens a été codifié par catégories, en retenant les arguments utilisés, l'énoncé des définitions, les normes et les valeurs, ainsi que le positionnement de chacun vis-à-vis des enjeux, ceci de façon à dégager des structures narratives (Mucchielli, 2004: 47-52). 
TABLEA U 1

Synthèse de la situation

\begin{tabular}{|c|c|c|c|c|c|c|}
\hline \multicolumn{7}{|c|}{ Éléments de la narration de la situation } \\
\hline & 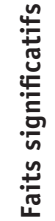 & 窇 & 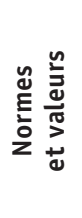 & 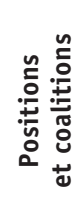 & 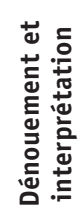 & 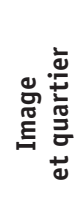 \\
\hline Acteur $\mathrm{A}$ & & & & & & \\
\hline Acteur B & & & & & & \\
\hline Acteur C & & & & & & \\
\hline
\end{tabular}

Cadre de référence inspiré de Mucchielli, 2004: 48.

À partir des narrations successives, nous avons constitué un récit commun, partagé de façon quasi unanime par les personnes interviewées, sans toutefois réduire toutes les informations. Des constats non partagés ont aussi été retenus, que nous qualifions de dissonants, non pas pour les disqualifier, ni même pour accentuer l'opposition au récit commun, mais simplement pour indiquer qu'ils apportent une interprétation qui s'écarte du courant principal et qui peut, occasionnellement, souligner une dimension oubliée ou contenir une formulation critique du processus. Ces constats dissonants n'ont été énoncés que par certains des acteurs rencontrés, voire par un seul d'entre eux.

Par la suite, le recours à l'approche transactionnelle permet de cerner les ajustements constants des acteurs, qui font évoluer la situation de départ, de même que les positions de chaque acteur sur les divers enjeux abordés, alors que ces changements ne sont que rarement énoncés de manière explicite. On essaie de cette façon de décortiquer la séquence des ajustements. Le schéma d'analyse de Blanc et al. (1998: 238) découpe le processus d'ajustement en six grandes étapes (tableau 2). Cette grille de lecture est utile pour éclairer les phases du processus de mobilisation, de concertation, de planification et d'apprentissage collectif qui découlent des situations observées. 
TA B LEA U 2

Schéma d'analyse de la transaction sociale

\begin{tabular}{|ll|}
\hline \multicolumn{2}{|l|}{ Étapes de l'analyse } \\
\hline 1- & Décrire la situation transactionnelle \\
\hline 2- & Situer les problèmes à résoudre \\
\hline 3- & Repérer les jeux d'acteurs \\
\hline 4- & Observer les effets sur le lien social \\
\hline $5-$ & Étudier les modifications du contexte, conséquences de l'agrégation des \\
& transactions \\
\hline $6-$ & S'interroger sur la construction de sens et l'émergence de nouvelles valeurs \\
\hline
\end{tabular}

Source: Blanc et al., 1998: 238.

Dans le cas du BUMP, nous avons voulu définir la nature de la situation dans laquelle se trouvaient les acteurs réunis par la concertation. Dans un premier temps, il s'agissait de déterminer les acteurs présents et les règles de procédures d'échange, puis la nature du problème, d'établir la position des acteurs et, enfin, de cerner les tensions, les compromis pratiques et les concessions possibles. Enfin, il s'agissait de reconnaître les ajustements identitaires, c'est-à-dire les changements des représentations individuelles qui sont liés à la transaction sociale, ainsi que ses effets sur la dynamique collective. L'étape finale vise à éclairer les changements en termes de valeurs et de construction de sens.

\section{La relance d'un projet de médiation urbaine: BUMP}

\section{La situation initiale}

La reconstitution de la situation initiale entourant le projet de médiation urbaine auprès des jeunes de la communauté noire du quartier de la Petite-Bourgogne a d'abord été faite à partir d'une entrevue avec le coordonnateur de la Table de quartier dont l'organisme fiduciaire est la Coalition de la Petite-Bourgogne. Celui-ci a rappelé qu'en 2003, lors du festival annuel du quartier, un jeune a été tué dans une altercation. Comme il s'agissait de l'élément culminant d'une vague de violence affectant les jeunes, les organismes communautaires du quartier se sont réunis afin de discuter de la question et la Coalition a lancé une démarche de réflexion sur la criminalité et la sécurité. Ce sont les membres de la communauté noire de la Petite-Bourgogne présents au conseil d'administration de la Coalition qui ont coordonné la réflexion sur les actions 
à entreprendre. Des responsables de la Ville de Montréal, de l'arrondissement et des élus ont été interpellés à la même époque, plus particulièrement le responsable des Services aux citoyens au Comité exécutif de la Ville de Montréal. Grâce à un fonds provenant du contrat de ville, la création d'un programme de médiation urbaine a été proposée.

Les versions diffèrent selon les interlocuteurs sur l'identité de l'organisme à l'origine du projet BUMP. Est-ce la Coalition ou des membres d'un organisme de la communauté noire? Quoi qu'il en soit, la négociation entre les acteurs communautaires réunis sous le chapeau de la Coalition et les partenaires institutionnels, notamment des représentants de la Ville, du Service de police, de la Société des transports de Montréal, a débouché sur la formation d'un nouvel organisme, le Black Family Support Group (BFSG). Celui-ci, formé de membres de la communauté noire anglophone, a présenté à la Ville un projet de médiation à l'hiver 2003-2004. La Coalition a accepté d'assumer le rôle de fiduciaire jusqu'à ce que le groupe soit incorporé en août 2004. À partir de ce moment, elle n'était plus officiellement associée au projet. Cependant, les deux organismes ont conservé des liens informels puisque des personnes participaient aux activités des deux groupes.

Cette première étape de la constitution du projet BUMP confirmait la Coalition dans son rôle dirigeant dans le réseau des acteurs sociaux présents dans le processus de concertation. Ce premier ajustement de la transaction sociale en cours avait pour but de recréer une plus grande proximité institutionnelle entre les différentes communautés du quartier et de produire une situation nouvelle, celle d'une action concertée auprès des jeunes et des familles.

Le projet mis en place comprenait, en effet, deux volets principaux: la médiation dans les espaces publics, tels les parcs, avec les jeunes et la médiation auprès des familles. L'objectif était d'atténuer les différences au sein de la communauté noire. Deux médiateurs ont été embauchés en plus d'un bénévole chargé de la coordination. Aux dires de toutes les personnes interviewées, le travail des médiateurs a été immédiatement apprécié et les tensions ont semblé s'amenuiser. Citoyens, intervenants et policiers ont souligné la qualité des interventions réalisées, ce qui a été confirmé d'ailleurs par une étude indépendante (Tremblay et al., 2006).

Des difficultés d'ordre administratif ont été observées à la fin 2005. Les règles habituelles de la gestion de programme ne semblaient pas être suivies. Des salaires impayés, le congédiement d'un médiateur et 
l'arrêt du fonctionnement normal de l'organisme ont amené la Coalition à intervenir, allant même jusqu'à avancer une importante somme d'argent pour couvrir les dépenses du projet, le temps que la situation soit régularisée auprès de la Ville, avance dont une partie est dorénavant comprise comme une contribution directe au projet. D'autres difficultés de fonctionnement ont été notées à l'été 2006, puis toutes les activités du programme ont cessé en octobre. À l'approche de l'été 2007, plusieurs citoyens ont fait pression pour la relance du projet: on anticipait la reprise des tensions dans les espaces publics du quartier. La population et les institutions se sont tournées vers la Coalition.

Ce deuxième moment de la transaction sociale entre la Coalition et le BFSG a révélé la nature du problème à résoudre, soit l'opposition entre deux conceptions de la vie associative. La Coalition, en venant à la rescousse du projet, pouvait revoir le mode de gestion particulier du projet BUMP et tenter d'y apporter des correctifs. Car, si tous s'entendaient sur la nécessité de la relance, le modèle organisationnel et le choix de l'organisme fiduciaire demeuraient litigieux. La communauté noire souhaitait reprendre sous le même mode de gestion plutôt informel, alors que les institutions et le bailleur de fonds exigeaient une structure plus conforme aux normes administratives. De plus, aucun organisme ne s'est porté d'emblée volontaire pour reprendre à son compte le projet.

\section{Le quartier, cadre urbain et espace de transition}

La Petite-Bourgogne conserve à ce jour l'image d'un quartier dangereux, lieu de criminalité et de trafic de drogue. Bien que cette perception soit démesurée, les répondants ont reconnu qu'il y persistait, au moment des discussions sur la relance du BUMP, une ambiance délétère de violence émanant surtout de la présence de bandes de jeunes prêts à adopter par mimétisme des comportements incivils. Ceux-ci n'étaient apparemment pas liés aux gangs de rue qui inspiraient la crainte dans d'autres quartiers montréalais, mais ils pouvaient exhiber certains de leurs comportements et attitudes.

Cette situation difficile vécue par les jeunes a été en maintes occasions reliée plus ou moins directement à la pauvreté, aux conditions matérielles d'existence, à la piètre qualité du cadre bâti, dues notamment aux interventions de renouveau urbain survenues durant les années 1950 et 1960. Tous ont rappelé comment la construction de l'autoroute 
Ville-Marie et des îlots Saint-Martin (un complexe HLM dans les années 1960) a bouleversé la vie de quartier et exacerbé les clivages entre les communautés qui s'y côtoient. Outre la communauté noire anglophone enracinée depuis plus d'un siècle, on compte aujourd'hui de nombreuses familles antillaises francophones et anglophones, sans compter des immigrants d'origines variées arrivés au pays plus récemment.

C'est donc un quartier d'accueil et de transition où la pauvreté s'ajoute aux difficultés d'intégration. On y a cité, lors des entretiens, d'importantes difficultés au chapitre de l'emploi, du logement et de l'éducation. Quant aux jeunes d'âge scolaire, «ils sont isolés dans une société étrangère ainsi que dans le système scolaire et les parents sont pris au dépourvu», indiquait un membre de la communauté. La médiatisation des problèmes sociaux de la Petite-Bourgogne a également contribué au sentiment de marginalité de la population. Enfin, il faut noter que les structures de concertation du quartier ne font pas suffisamment place aux communautés d'immigration récente, souvent anglophones, qui semblent plus particulièrement affectées par les problèmes des jeunes. Cela représente un défi qui préoccupe les membres de la Coalition.

\section{Les acteurs présents}

La Table intersectorielle, sous la responsabilité de la Coalition de la Petite-Bourgogne, est de type «quartier en santé», c'est-à-dire qu'elle n'est pas composée uniquement du secteur communautaire. Elle est intersectorielle et multiréseau. Régie par un conseil d'administration où siègent une majorité de représentants du monde communautaire (six en 2007-2008), trois résidents et deux représentants institutionnels, la composition de la Table est organisée en collèges où les représentants institutionnels jouent un rôle important. Ceux-ci proviennent, par exemple, de la Ville de Montréal, de l'Arrondissement, du Centre de santé et des services sociaux ou du CLSC. La Table rejoint l'ensemble des instances communautaires et institutionnelles qui touchent à sa mission, sans chercher à les faire entrer dans une structure intégrée ou hiérarchisée. En d'autres termes, la Table de concertation du quartier réunit des acteurs issus de la société civile et des institutions publiques, à l'intérieur d'une instance démocratique, partageant le même espace de délibération, sans parvenir à éliminer les avantages reliés à la position de pouvoir qu'occupent les opérateurs publics. Sa mission se concentre sur les questions de développement urbain et de développe- 
ment social. La Table est aussi formée de six comités sectoriels, sur la jeunesse, la sécurité alimentaire, l'habitation, l'environnement et la sécurité, le tissu social et l'employabilité. À son endroit, les institutions de la communauté noire ont adopté une posture distante et critique puisqu'elles la considèrent comme trop bureaucratique et regroupant des non-résidents de la Petite-Bourgogne. Bien que plusieurs membres de cette communauté participent à la Table à titre individuel, le maintien des liens entre les structures de concertation et la communauté noire est un enjeu permanent. Cette communauté possède, en fait, des organisations distinctes, parfois émanant des églises, certaines décrites comme de type grassroots, au fonctionnement informel.

Dans le cas du BUMP, les services de police du quartier, les groupes qui travaillent auprès des jeunes et des résidents ont joué un rôle important dans le processus de sa formation puis dans la tentative de relance en 2007. Soit dit en passant, son approche correspond à celle décrite dans le Plan québécois qui vise l'augmentation «de la capacité d'intervention des milieux municipal et communautaire à prévenir l'adhésion des jeunes aux gangs de rue» (ibid.: 18). Celle-ci semble même faire consensus (Mourani, 2006), si bien que le Service de police de la Ville de Montréal l'a reprise à son compte en privilégiant la concertation et les relations avec la communauté (SPVM, 2008). Soutien à la communauté, mobilisation des intervenants et prévention à l'adhésion des jeunes aux gangs sont certainement les maîtres mots du Plan québécois comme du projet BUMP.

\section{Enjeux: les facteurs de réussite et d'échec}

Le projet BUMP croise les enjeux de la sécurité avec ceux des relations intercommunautaires et intergénérationnelles. L'enjeu des interactions entre les jeunes et les services policiers est aussi compris comme capital. Le maintien d'une sorte de vouloir-vivre-ensemble à l'intérieur du quartier demeure une préoccupation et cela passe par la concertation et une forme ou l'autre d'empowerment local. «Avec le BUMP, le quartier donne l'impression de se prendre en main pour la première fois», a remarqué un représentant des forces de l'ordre, au point que d'autres localités se seraient intéressées aux développements survenus en la matière dans la Petite-Bourgogne. C'était une initiative conçue et portée par des membres de la communauté noire anglophone et les médiateurs choisis pour travailler sur le terrain étaient connus des gens 
du quartier. Les deux médiateurs se sont investis sans compter dans la résolution de problèmes (horaires atypiques et longues heures impayées) et ils ont vaincu la méfiance et le risque d'être perçus comme des «stools» par les jeunes du quartier. Lancrage local et les origines des intervenants ont assuré le succès du BUMP sur le terrain.

Toutes les personnes associées au processus de relance du BUMP reconnaissaient que l'échec du projet était de nature administrative: le BFSG n'aurait jamais mis en place une structure organisationnelle définie; le suivi administratif et comptable aurait été déficient; les règles de gestion de la Ville n'ont pas été suivies. Selon l'ancienne directrice de l'organisation, par contre, le projet aurait été sous-financé et le soutien de la Ville était insuffisant. Beaucoup de travail a été accompli de façon bénévole mais l'intérêt s'est dissipé : la participation a diminué et la relève a manqué.

Par ailleurs, la communauté appréciait énormément la latitude accordée aux médiateurs qui intervenaient dans toutes sortes de situations. Mais cette liberté d'action dénotait, selon plusieurs, un laxisme sur le plan de la supervision du personnel. Les représentants de plusieurs institutions estimaient que les médiateurs ont outrepassé leur mandat dans certains cas et sont intervenus dans des situations qui commandaient plutôt la référence à d'autres instances (police, DPJ, etc.).

Pour résumer les difficultés de l'organisation chargée du projet BUMP, on citait des problèmes administratifs et financiers ainsi qu'une ambiguiité persistante du rôle des intervenants dans le projet. D'autres ont répliqué que le fonctionnement informel n'a jamais posé de problèmes; ils ont mis en cause la Ville qui n'était ni préparée ni organisée pour administrer un tel type de projet. La représentante d'un autre organisme du quartier déplorait que l'échec de ce projet soit perçu comme l'échec de toute la communauté noire.

\section{Négociations et points de blocage}

En dépit des problèmes vécus au sein de l'organisation, les intervenants du quartier et tous les représentants de la communauté noire souhaitaient le retour du projet BUMP à l'été 2007. La Ville, comme le Service de police, a considéré l'initiative comme porteuse de solutions et comptait sur sa reprise en 2007. En l'absence du projet, les policiers envisageaient une recrudescence importante de la criminalité juvénile et le retour à des rapports plus tendus avec les résidents. 
Au moment des discussions sur la relance, les clivages observés concernaient premièrement la désignation de l'organisme fiduciaire chargé du projet et, deuxièmement, le modèle organisationnel à privilégier. Plusieurs représentants de la communauté noire, principalement des résidents siégeant au conseil d'administration de la Coalition, réclamaient le retour des activités selon le même modèle, celui proche du type grassroots. Ils estimaient avoir été bien servis durant la première phase et peu leur importait que des irrégularités administratives ou d'un autre ordre soient survenues. Cela étant, le bailleur de fonds exigeait qu'un suivi administratif et comptable plus rigoureux soit établi au préalable. L'absence d'une hiérarchie décisionnelle et le caractère informel des opérations pouvaient paraître intéressants sur le plan de l'intervention mais ils compliquaient la gestion et ouvraient la porte aux fautes professionnelles.

La proposition selon laquelle la Coalition devait administrer le projet de manière intérimaire est devenue la seule envisageable aux yeux de plusieurs des personnes rencontrées lors des entretiens. Malgré l'absence d'alternative, cette proposition a été reçue froidement par ceux qui croyaient que la Coalition ne disposait pas de l'expertise technique nécessaire. En outre, une éventuelle prise de contrôle du BUMP par la Coalition risquait d'exacerber les tensions existantes entre la communauté noire et les partenaires de la concertation de quartier.

\section{L'interprétation du dénouement}

En l'absence d'alternative provenant d'organismes du quartier, les membres du conseil d'administration de la Coalition ont accepté que l'organisation reprenne la direction du projet pour l'été 2007. Cet arrangement perdurait encore à l'hiver 2009. La Coalition devait assurer le suivi administratif sur une base intérimaire en s'adjoignant une équipe de médiateurs urbains chargée du travail de terrain. Ce dénouement est interprété à travers un cumul de récits compilés dans le tableau synthèse des différents entretiens (tableau 3) qui départage ce qui est commun de ce qui est spécifique et que l'on qualifie de dissonant.

\section{Entre autonomie et rigueur administrative}

Le projet BUMP est né dans un moment de crise afin de répondre au sentiment d'insécurité qui s'était propagé dans le quartier. Il est devenu 
une priorité des institutions publiques, le Service de police compris, au point d'être jugé indispensable. La qualité du travail de terrain n'a jamais été mise en cause. Plutôt, ce sont des problèmes de nature administrative qui l'ont discrédité. Plusieurs lui ont reproché de ne pas avoir bien établi clairement le rôle de chacun et, finalement, d'avoir fonctionné en vase clos. Le BUMP n'a pas permis de jeter des ponts entre toutes les communautés de la Petite-Bourgogne. La question de la reconnaissance de l'autonomie organisationnelle de la communauté noire avec, en contrepartie, la représentativité des structures de quartier instituées autour de la Table formaient le nœud des obstacles de la relance. Il s'agissait d'établir une proximité organisationnelle entre les partenaires de la Table, Ville et SPUM compris, et les acteurs en marge de la concertation, mais seuls capables de traiter d'un problème aussi sensible que les jeunes et les gangs de rue. Pour ce faire, la Coalition de la Petite-Bourgogne devait trouver un terrain d'entente avec les représentants de la communauté noire. Le débat entourant la relance du BUMP n'a pas été celui de la reddition de comptes, mais bien celui de choisir entre deux modèles organisationnels, soit le modèle institutionnel, soit le modèle dit de grassroots plus informel et intuitif. Ces deux «philosophies» de la vie associative se distinguent également par la façon de transiger avec les institutions publiques, dont la Ville et le Service de police, comprises comme des partenaires par les uns et comme représentants du pouvoir établi sur lesquels exercer des pressions politiques par les autres. En ce sens, la transaction sociale qui recouvre le processus de relance du BUMP porte sur l'adaptabilité du modèle partenarial développé à Montréal depuis plusieurs années et officialisé avec le programme de soutien aux Tables de quartier. Elle n'altère ni la représentativité de la Coalition ni sa capacité d'agir, tout en introduisant une marge d'autonomie dans la gestion d'un projet particulier: le BUMP.

Deux régimes de valeurs sont exprimés à l'intérieur des différents récits, celui des partenaires sociaux de la concertation de quartier et celui des représentants des organismes de la communauté noire. Ils ne peuvent pas toutefois être tenus comme totalement opposés; ils révèlent des postures différentes même si des valeurs communes les émaillent. On serait même tenté de les interpréter comme coprésents, voire nécessaires l'un à l'autre. Les institutions ont avancé le principe d'arrimage de la communauté noire au réseau des organismes du quartier en insistant sur la reddition des comptes, pour défendre finalement des règles 
TABLEAU 3

\section{LE BUMP: Une synthèse de la situation}

\begin{tabular}{|c|c|c|c|c|c|}
\hline \multicolumn{6}{|c|}{ Éléments de la narration de la situation } \\
\hline & Faits significatifs & Enjeux et arguments & Normes et valeurs & Positions et coalitions & $\begin{array}{l}\text { Dénouements } \\
\text { et interprétations }\end{array}$ \\
\hline $\begin{array}{l}\text { Constats } \\
\text { partagés }\end{array}$ & $\begin{array}{l}\text { Situation de crise (violence, } \\
\text { insécurité). } \\
\text { Soutien institutionnel au } \\
\text { BUMP. } \\
\text { Bilan positif du projet } \\
\text { (travail de terrain). } \\
\text { Difficultés budgétaires et } \\
\text { administratives. } \\
\text { Volonté de relance. } \\
\text { La Ville a soutenu le BUMP } \\
\text { et proposé un fonds. }\end{array}$ & $\begin{array}{l}\text { Urgence d'agir. } \\
\text { Sécurité. } \\
\text { Établir de bonnes relations } \\
\text { entre les jeunes, la police, } \\
\text { les résidants. } \\
\text { Répondre aux besoins des } \\
\text { jeunes Noirs. } \\
\text { Concilier les exigences } \\
\text { administratives et } \\
\text { budgétaires et le mode } \\
\text { de grassroots. }\end{array}$ & $\begin{array}{l}\text { Confiance; consultation; } \\
\text { participation. Transparence } \\
\text { et dialogue. } \\
\text { Agir sur les causes de la } \\
\text { violence. } \\
\text { Prévoir à long terme. } \\
\text { Solidarité des partenaires, } \\
\text { des groupes et des } \\
\text { résidants. } \\
\text { L'engagement de la } \\
\text { communauté noire. }\end{array}$ & $\begin{array}{l}\text { La Ville, la police et la CPB } \\
\text { s'entendaient pour la relance. } \\
\text { La Coalition doit s'assurer que } \\
\text { les services sont rendus. } \\
\text { Le BFSG était isolé. } \\
\text { Beaucoup d'attention } \\
\text { accordée à la CN par la Ville. } \\
\text { Arrimage institutionnel } \\
\text { difficile. }\end{array}$ & $\begin{array}{l}\text { La CPB a fait le travail pour } \\
\text { préparer la relance. } \\
\text { Beaucoup de travail reste à } \\
\text { faire: } \\
\text { Mieux encadrer le travail des } \\
\text { médiateurs; } \\
\text { rétablir le lien de confiance } \\
\text { entre les jeunes et la police; } \\
\text { clarifier les rôles (gestion / } \\
\text { terrain) et le fonctionnement } \\
\text { du BUMP. }\end{array}$ \\
\hline $\begin{array}{l}\text { Constats } \\
\text { dissonants }\end{array}$ & $\begin{array}{l}\text { Ambiguïté des rôles des } \\
\text { partenaires. } \\
\text { Le BUMP était sous-financé. } \\
\text { La représentativité de la } \\
\text { CPB était inadéquate. } \\
\text { Le BUMP est défini comme } \\
\text { un grassroots. }\end{array}$ & $\begin{array}{l}\text { Autonomie des institutions } \\
\text { de la communauté noire. } \\
\text { Besoin d'un leadership local } \\
\text { propre à la communauté } \\
\text { noire. } \\
\text { Risque de limiter la portée } \\
\text { du BUMP en le laissant sous } \\
\text { la gestion des institutions. }\end{array}$ & $\begin{array}{l}\text { Pas d'intérêts divergents au } \\
\text { sein du projet. } \\
\text { Besoin de rebâtir la } \\
\text { crédibilité. } \\
\text { Briser la méfiance. } \\
\text { On doit protéger l'informa- } \\
\text { tion recueillie sur le } \\
\text { terrain. }\end{array}$ & $\begin{array}{l}\text { Stratégies différentes des } \\
\text { acteurs présents. } \\
\text { La Ville a des intérêts } \\
\text { particuliers, dont celui d'assu- } \\
\text { rer le contrôle social. } \\
\text { La participation de la } \\
\text { population était limitée dans } \\
\text { le projet. }\end{array}$ & $\begin{array}{l}\text { Trop de considérations } \\
\text { politiques. } \\
\text { La communauté noire tient à } \\
\text { sa culture organisationnelle } \\
\text { propre. } \\
\text { La CPB n'a pas l'expertise pour } \\
\text { diriger le BUMP. } \\
\text { Les conditions de la Ville } \\
\text { risquent de nuire au BUMP. }\end{array}$ \\
\hline $\begin{array}{l}\text { Images du } \\
\text { quartier }\end{array}$ & $\begin{array}{l}\text { Dégradation du cadre } \\
\text { urbain. } \\
\text { Quartier d'accueil et } \\
\text { d'immigration. } \\
\text { Tensions entre la police et } \\
\text { la communauté noire. } \\
\text { Marginalisation de la } \\
\text { communauté noire. }\end{array}$ & $\begin{array}{l}\text { Lutter contre les clivages } \\
\text { sociaux, l'insécurité, la } \\
\text { ségrégation. } \\
\text { L'espace de médiation des } \\
\text { problèmes à construire. } \\
\text { Manque de sentiment } \\
\text { d'appartenance au quartier. }\end{array}$ & $\begin{array}{l}\text { La nécessaire réparation du } \\
\text { quartier. } \\
\text { L'identité. } \\
\text { L'intégration intercommu- } \\
\text { nautaire. } \\
\text { Prise en charge des } \\
\text { problèmes par les groupes } \\
\text { et les associations du } \\
\text { quartier. }\end{array}$ & $\begin{array}{l}\text { Deux modèles organisation- } \\
\text { nels qui s'opposent: } \\
\text { partenariat institutionnalisé } \\
\text { et grassroots informel. }\end{array}$ & $\begin{array}{l}\text { Un arrangement était possible } \\
\text { mais la confiance doit être } \\
\text { rétablie entre les partenaires } \\
\text { sociaux et la communauté } \\
\text { noire. } \\
\text { La figure du quartier est une } \\
\text { juxtaposition de communau- } \\
\text { tés et d'acteurs en interac- } \\
\text { tion. }\end{array}$ \\
\hline
\end{tabular}


de gestion uniformes. Dans la foulée, ses représentants ont mis de l'avant le principe de démocratisation de la vie associative et, du même souffle, souligné l'hermétisme des organismes informels de type grassroots. Ils auraient souhaité l'adoption de formes de participation et de consultation largement ouvertes sur l'ensemble de la communauté. Ce faisant, la prétention était de favoriser une meilleure intégration des Noirs au réseau institutionnel du quartier. Ce premier régime de valeurs est ancré dans le fonctionnement des administrations publiques et, de la même façon, des organismes locaux engagés dans des programmes publics normés. Il reste toutefois insuffisant pour aborder une question aussi sensible et cruciale que celle des jeunes et de la sécurité urbaine puisqu'il se limite aux formes de gestion et de participation. Le deuxième régime de valeurs se rapporte au contenu même du projet BUMP et il valorise l'action, le travail de terrain, la confiance, le contact entre les médiateurs et les jeunes. Les acteurs du milieu ont accordé une grande attention à l'expérience des résidants en situation d'insécurité, au travail délicat des médiateurs, au niveau de confiance que les jeunes ressentent vis-à-vis des médiateurs et des policiers. On pourrait coiffer ces deux propositions issues des deux régimes de valeurs par les termes de la légitimité organisationnelle d'une part et de la capacité d'agir d'autre part. Ils ont été mentionnés sous différents vocables, par les uns et les autres, non pas pour exprimer une négation d'un autre point de vue, mais plutôt pour marquer une position dans une recherche de compromis.

La recherche de compromis durant cette phase 2007 de relance du BUMP était manifeste du seul fait que toutes les personnes rencontrées étaient convaincues de la nécessité du projet. Une véritable coalition sociale, dépassant le seul fait de la communauté noire, construite à l'échelle de la Petite-Bourgogne, a revendiqué la poursuite du BUMP. Des membres de la Coalition de la Petite-Bourgogne, les représentants des institutions publiques et du SPVM, des organismes communautaires ont participé à cette coalition. Autour du projet BUMP, des acteurs sociaux ainsi que des membres de la communauté noire ont formé un ensemble distinct qui, bien qu'en contact avec la Coalition de la PetiteBourgogne, a maintenu une distance et a prétendu à une autonomie propre. Le rapport de force engagé entre les deux ensembles paraissait à première vue inégal, les acteurs sociaux institutionnalisés bénéficiaient d'un avantage comparatif certain, en raison notamment de la présence du bailleur dans un partenariat constitué et durable, mais les membres 
de la communauté noire pouvaient revendiquer le lien au terrain et à l'action que ne possédaient pas les premiers.

\section{La reconstruction de l'image du quartier}

Dans la lutte à la délinquance juvénile, et face au risque que font peser les gangs de rue, le quartier est décrit sous les traits de la désorganisation sociale et des inégalités sociales. Il est aussi caractérisé par la présence d'un ensemble institutionnel solidaire et chargé de la régulation sociale. La densité du réseau associatif du quartier et les partenariats noués avec les organismes publics sont compris comme des conditions nécessaires à toute démarche en vue d'apporter des réponses aux situations problématiques. C'est sous cette double image, celle du quartier défavorisé et de l'espace de solidarité, que le processus de relance du BUMP est engagé. Cela a été l'occasion pour les acteurs d'exiger la réparation des erreurs du passé en matière urbanistique, l'amélioration des relations intercommunautaires et la prise en charge par les intervenants locaux des problèmes du quartier. Les réponses devraient émaner de ce travail de concertation qui met en scène le tissu dense d'organismes et de groupes, ancrés localement, dont le mandat est de définir les enjeux et de négocier des pistes d'action. Ce mandat exige de rejoindre et de faire participer toutes les parties du milieu. En reconnaissant que la concertation de quartier a d'abord eu pour objectif d'intégrer toutes les communautés du territoire, notamment celles qui, à l'instar de la communauté noire, se sentaient marginalisées, le processus de concertation a d'abord visé à faire concorder l'image multiculturelle du quartier et la représentativité du réseau organisationnel chargé de parler en son nom. La relance du BUMP a permis de renouer le dialogue intercommunautaire sans régler toutes les tensions entre les partenaires sociaux et les représentants de la communauté noire. Le processus a démontré, par contre, qu'il était possible de parvenir à un arrangement avec la communauté noire, fût-il ponctuel, et ainsi recomposer la mosaïque du quartier en l'incluant. Il a également conduit à un arrangement entre les deux régimes de valeurs identifiés dans le débat, la formation d'une légitimité organisationnelle reconnue et le soutien à la capacité d'agir sur le terrain. 


\section{Les termes de la transaction}

La relance du projet BUMP a porté d'abord et avant tout sur les formes organisationnelles qui devaient régir le projet, incluant les règles et les procédures administratives ainsi que le mode de fonctionnement de l'organisme porteur. Par là, il s'agissait de refonder le lien social entre les acteurs des différentes communautés, dont ceux de la communauté noire. En cherchant à identifier l'organisation idéale pour gérer le BUMP, le rôle et la nature même de la Table de quartier, la Coalition de la Petite-Bourgogne en l'occurrence, étaient aussi remis en question. La légitimité et la représentativité de la Table ont été discutées. Les acteurs partenaires de la Table, dont les institutions publiques, ont dû accepter de transiger à propos d'un projet discrédité au regard des règles administratives de l'administration publique. L'espace de la concertation a été ainsi étendu vers des acteurs que l'on peut qualifier de distants et ne partageant pas le modèle partenarial préconisé par la Table. Le projet BUMP a donc constitué, pour la Coalition, une occasion de démontrer sa capacité d'adaptation et d'affirmer sa volonté d'accroître sa représentativité. La Coalition devait à la fois faire preuve de leadership, de capacité de rassemblement en orchestrant les négociations de la relance du BUMP et consolider ses liens avec la communauté noire.

D'une certaine façon, les modes habituels de régulation sociale, que l'administration publique, la police ou les Églises ont incarnés de tout temps, sont apparus transformés à la lumière des discussions sur la relance du BUMP. Les pratiques de partenariat et de participation, appliquées aux tensions soulevées par le projet, ont permis une légitimation accrue de l'action publique dans le quartier. Le dialogue renouvelé entre les différents acteurs a été reconnu comme équitable. Il a conduit à des ajustements successifs et débouché sur ce que nous qualifions de compromis de coexistence entre les différentes communautés. La transaction sociale s'est ici traduite par le passage d'une forme de coopération conflictuelle à un arrangement institutionnel acceptable pour toutes les parties.

Sur le plan pratique, le problème à résoudre a consisté à réconcilier différentes logiques institutionnelles et communautaires, en partageant finalement le travail en deux unités, l'une gestionnaire, l'autre chargée du terrain. Certes, ce compromis de coexistence était compris comme transitoire. Il laissait une grande autonomie d'action aux intervenants sur le terrain, ce qui correspondait aux demandes exprimées par les 
représentants de la communauté noire. Il confirmait en quelque sorte la capacité transactionnelle de la Coalition, qui devait se révéler comme un espace de conciliation entre les institutions publiques représentées à la Table et les formes diverses de la vie associative du quartier, incluant celles plus informelles, voire celles qui s'expriment par l'entremise des personnes plutôt que par des structures instituées et normées. Ce faisant, le réseau associatif et institutionnel réuni par la Table pouvait recomposer l'image du quartier comme espace d'intervention et de solidarité. Il le pouvait d'autant plus que la transaction sociale réalisée donnait un sens et une portée à la volonté de vivre ensemble que suppose l'image de quartier multiethnique accolée à la Petite-Bourgogne.

Le dénouement de la situation a laissé ouvert le système d'interactions entre les institutions et la communauté noire, les représentants institutionnels et les individus concernés par la situation. Il témoigne d'un apprentissage collectif, celui d'arrimer les agents de la concertation et les intervenants qui sont sur le terrain, faisant en sorte que le consensus qui s'est formé dépasse le mandat de la Coalition pour rejoindre les jeunes, les médiateurs, les policiers et les résidents. La transaction qui opposait des principes bien établis, de part et d'autre, a porté sur le passage à l'action. Les principes de chacun des deux univers, institutionnel et informel, paraissent avoir été respectés. Certes, on serait tenté d'interpréter le dénouement comme la formation d'un grassroots sous surveillance. N'est-ce pas plutôt un ajustement qui injecte un sens nouveau à la concertation intersectorielle et au partenariat interréseau en mettant de l'avant la primauté de l'action et du terrain? En s'efforçant de maintenir les liens avec la communauté noire et de rétablir la proximité organisationnelle entre les réseaux et les communautés, de former la compétence des acteurs à soutenir l'espace de proximité, la Coalition s'est replacée en situation transactionnelle au nom de son mandat social et territorial. La transaction portait aussi sur l'image du quartier: la présence de la communauté noire dans la Petite-Bourgogne devait être réaffirmée au sein de la Coalition.

En définitive, la lutte aux gangs de rue exige, du moins le volet qui fait appel à la concertation et à la mobilisation des acteurs locaux, une réelle capacité de réflexivité autour de l'action communautaire territorialisée, de façon à penser que les stratégies d'intervention et le modèle organisationnel privilégié soient adaptés à la réalité du quartier et puissent rendre compte des réalités propres aux communautés qu'on veut rejoindre. 


\section{Références}

Asher, F. (1998). La fin des quartiers. In N. Haumont (ed.) L'urbain dans tous ses états (183-201). Paris: L'Harmattan.

Blanc, M. (1992). Pour une sociologie de la transaction sociale. Paris: L'Harmattan.

Blanc, M., Pineau, G., \& Frenet, M.-F. (1998). Les transactions aux frontières $d u$ social. Formation, travail social, développement local. Lyon: Chronique Sociale.

Block, R., Galary, A., \& Brice, D. (2007). The Journey to Crime: Victims and Offenders Converge in Violent Index Offences in Chicago. Security Journal, 20 (2), 123-137.

Blumer, H. (1969). Symbolic Interactionism, Perspectives and Method. Englewood Cliffs NJ: Prentice-Hall.

Burgess, E. W. (1925). Can Neighbourhood Work Have a Scientific Basis?, In R. E. Park \& E. W. Burgess, (eds.), The City (142-155), Chicago: University of Chicago Press, éd. 1967.

Chalas, Y. (1997). Le déclin du quartier. Urbanisme, 297, 49-51.

Charbonneau, J. (1998). Lien social et communauté: quelques questions préalables. Liens social et politique, 39, 115-126.

Giddens, A. (1994). Les conséquences de la modernité, Paris: L'Harmattan.

Gilly, J.-P., \& Lung, Y. (2005). Proximité, secteurs, territoires. Cahiers du GRES, $\mathrm{n}^{\circ} 9$, en ligne. http//www.gres.so.org.

Goffman, E. (1974). Les rites d'interaction. Paris: Minuit.

Gouvernement du Québec (2007). Plan d'intervention québécois sur les gangs de rue, 2007-2010. Québec: Ministère de la Sécurité publique.

Gregmeyer, Y. (2007). Le quartier des sociologues. In J.-Y. Authier, M.-H. Bacqué \& F. Guérin-Pace (eds.), Le quartier. Enjeux scientifiques, actions politiques et pratiques sociales (21-31). Paris: La Découverte.

Habermas, J. (1992). L'espace public. Paris: Payot.

Joseph, I., \& Quéré, L. (2003). L’organisation sociale de l'expérience, Multitudes, http://multitudes.samizdat.net/L-organisation-sociale-de-1.html.

Milburn, P. (1999). Criminalité et crime. In A. Akoun \& P. Ansart (eds.), Dictionnaire de la sociologie (122). Paris: Seuil.

Mourani, M. (2006). La face cachée des gangs de rue. Montréal: Les Éditions de l'Homme.

Mucchielli, A. (2004). Dictionnaire des méthodes qualitatives en sciences sociales. Paris: Armand Colin.

Rémy, J. (1996). La transaction, une méthode d'analyse: contribution à l'émergence d'un nouveau paradigme. Environnement et Société, 17, 9-31.

Sénécal, G., Cloutier, G., \& Herjean, P. (2008). Le quartier comme espace transactionnel, l'expérience des Tables de concertation de quartier à Montréal. Cabiers de Géographie du Québec, 52 (146), 191-214.

SPVM (2008). Gangs de rue, site internet, consulté en décembre 2008, http:// www.spvm.qc.ca/fr/service/1_4_3_gangs-de-rue.asp. 
Stoessel-Ritz, J. (2005). Les lieux culturels comme espace public: transactions sociales pour un modèle du vivre-ensemble. Revue des Sciences sociales, 33, 122-129.

TIichit, L. (2003). Gangs juvéniles et construits ethniques dans le contexte américain, Criminologie, 36 (2), 57-68.

Trascher, F. M. (1927). The gang. A Study of 1313 gang in Chicago. Chicago: University of Chicago Press.

Tremblay, C. Jaccoud, M., \& Dupont, B. (2006). Projet de médiation urbaine de la Petite-Bourgogne. Montréal: Centre international de criminologie comparée.

Ville de Montréal (2006). Un quartier où il fait bon vivre, L'initiative montréalaise de soutien au développement social. Montréal: Comité tripartite Ville de Montréal, Direction de la santé publique de Montréal Centre et Centraide.

Wellman, B. (2001). Physical Place and Cyberplace: The Rise of Personalized Networking. International Journal of Urban and Regional Research, 25, 227252.

Wellman, B., \& Leighton, B. (1979). Networks, Neighborhoods and Communities, Urban Affairs Quarterly, 14, 363-90.

Whyte, W, F. (1943). Street Corner Society: The Social Structure of an Italian Slum. Chicago: University of Chicago Press.

Wirth, L. (1980). Le ghetto. Grenoble: Presses Universitaires de Grenoble.

ABSTRACT - In this paper, we analyse the revival process of an urban mediation project intended specifically at Black youth living in Montréal's Little Burgundy neighbourhood. The project known as BUMP (Burgundy Urban Mediation Project) is consistent with wider Québec tendencies that seek to facilitate a dialogue between social partners. The analysis of this process shows the conditions under which the neighbourhood is evolving and the emerging organizational models of social networks. The aim of our study is to consider the way social transactions take place between social partners and representatives of Black community organizations in order that urban mediation projects can be revived. Obstacles and opportunities for fostering such a participatory and partnership initiative are identified. The study concludes with a discussion on the position held and the role played by representatives of disenfranchised communities taking part in local cooperative bodies.

KEY WORDS - Street gangs, urban mediation, social transaction, Little Burgundy, Montréal.

RESUMEN - El presente artículo analiza el proceso de relanzamiento de un proyecto de mediación urbana orientado a jóvenes negros del barrio La Petite-Bourgogne de Montreal. Denominado BUMP (Burgundy Urban Mediation Project), el proyecto sigue las orientaciones generales del gobierno de la provincia de Quebec en materia de prevención del pandillaje basadas en la concertación entre diferentes actores sociales del propio barrio. El análisis de este proceso de relanzamiento pone de manifiesto las condiciones específicas del barrio y los modelos organizativos de sus redes sociales. El estudio analiza por otra parte la forma en que se efectuaron las transacciones sociales 
entre los diversos actores sociales y los representantes de organizaciones de la comunidad negra y que permitieron relanzar este proyecto de mediación urbana. Se analizan también los obstáculos y los factores que favorecieron dicha iniciativa de colaboración entre los participantes. El estudio concluye con una discusión sobre las posiciones de los representantes de las comunidades marginadas y su papel en las organizaciones de cooperación locales.

PALABRAS CLAVE - Pandillas callejeras, mediación urbana, barrio, transacciones sociales, Petite-Bourgogne, Montreal. 